\title{
Algunas consideraciones acerca del embarazo precoz en estudiantes universitarios. Provincia Manabí, Ecuador
}

\author{
Some considerations about early pregnancy in university students. Manabí \\ Province, Ecuador
}

\section{Algumas considerações sobre gravidez precoce em estudantes universitários. Província de Manabí, Equador}

Aleyma de Lourdes Vázquez-Medina ${ }^{\mathrm{I}}$

aleymalvm@gmail.com

Iván Gil-Jiménez ${ }^{\text {II }}$

ivan.gil69@yahoo.es

Luís Leandro Simón-Cedeño ${ }^{\text {III }}$

luisleandrosimon2015@gmail.com

Recibido: 10 de enero de 2019 *Aceptado: 15 de marzo de 2019 * Publicado: 05 de abril de 2019

I Especialista de Primer Grado en Medicina General Integral, Especialista de Primer Grado en Cirugía General, Doctora en Medicina. Docente de la Universidad Laica Eloy Alfaro de Manabí, Manta, Ecuador.

II Especialista de Primer Grado en Cirugía General, Doctor en Medicina Docente de la Universidad Laica Eloy Alfaro de Manabí, Manta, Ecuador.

III Especialista de Primer Grado en Ortopedia y Traumatología, Doctor en Medicina. Docente de la Universidad Laica Eloy Alfaro de Manabí, Manta, Ecuador. 


\section{Resumen}

La temática del embarazo en la adolescencia resulta de gran importancia en la actualidad debido a la trascendencia de este grupo etáreo para el desarrollo y su impacto en la población mundial, particularmente en América Latina y el Caribe. A tales efectos se realizó una revisión bibliográfica exhaustiva para exponer algunos aspectos relacionados con este asunto (definición, la salud sexual y reproductiva en los adolescentes, determinantes de riesgo para el inicio de la actividad sexual temprana y los implicados con el embarazo,) y se puntualizó el embarazo en la adolescencia en el Ecuador evidenciados en resultados de investigaciones en provincia de Manabí.

Palabras claves: Adolescencia; embarazo; repercusión biopsicosocial; labor educativa.

\section{Abstract}

The issue of pregnancy in adolescence is of great importance nowadays due to the importance of this age group for development and its impact on the world population, particularly in Latin America and the Caribbean. To this end, an exhaustive bibliographical review was carried out to expose some aspects related to this issue (definition, sexual and reproductive health in adolescents, determinants of risk for the initiation of early sexual activity and those involved with pregnancy), and pointed out the pregnancy in adolescence in Ecuador evidenced in results of investigations in the province of Manabí.

Keywords: Adolescence; pregnancy; biopsychosocial repercussion; educational work.

\section{Resumo}

A questão da gravidez na adolescência é de grande importância hoje em dia, devido à importância dessa faixa etária para o desenvolvimento e seu impacto na população mundial, particularmente na América Latina e no Caribe. Para tanto, foi realizada uma revisão bibliográfica exaustiva para expor alguns aspectos relacionados a essa questão (definição, saúde sexual e reprodutiva em adolescentes, determinantes de risco para o início da atividade sexual precoce e aqueles envolvidos com a gravidez), e apontou a gravidez na adolescência no Equador evidenciada em resultados de investigações na província de Manabí. 
Palavras-chave: Adolescência; gravidez; repercussão biopsicossocial; trabalho educativo

\section{Introducción}

La palabra adolescencia deriva del significado latino del verbo adoleceré («crecer»), que se identifica con cambios importantes en las esferas biológicas, psicológicas y sociales del ser humano. Se conceptualiza como un fenómeno cultural y social, por lo tanto, sus límites no se asocian fácilmente a características físicas. (Centre for Disease Control and Prevention (CDC))

La Organización Mundial de la Salud (OMS) la define como: «Etapa que transcurre durante el segundo decenio de la vida de los seres humanos, es decir, entre los 10 y los 19 años, existiendo una diferencia en su etapa temprana (10-14 años) y la tardía (15-19 años). Además refiere que es el período de la vida en el cual el individuo adquiere la capacidad reproductiva, transita los patrones psicológicos de la niñez a la adultez y consolida la independencia socio económica. (American Social Health Association. (Asociación Estadounidense de Salud Social). , 2009).

El adolescente interactúa en forma permanente con su núcleo social primario: la familia y la comunidad que lo rodea. Por lo tanto, las acciones dirigidas a mantener o recuperar la salud integral del adolescente deben tener un enfoque participativo, familiar y comunitario, con énfasis en la prevención primordial y primaria, para promover estilos de vida saludables._(Vaillant Correoso, Dandicourt, \& Mackensie, 2012)

La educación es una variable clave en la explicación y la transformación de los fenómenos relacionados con la salud reproductiva. Es un instrumento importante para lograr la conservación y el restablecimiento de la salud individual y de las comunidades, ya que ejerce su influencia sobre los conocimientos, criterios, convicciones, motivaciones y actitudes de los individuos. La educación sexual es por todo, un proceso vital mediante el cual se adquieren y transforman de manera formal e informal, conocimientos, actitudes y valores respecto a la sexualidad en todas sus manifestaciones, que van desde los aspectos biológicos hasta los relacionados con la reproducción, el erotismo, la identidad y las representaciones sociales. (Sexo y Salud,2005) 
La población mundial de adolescentes ha ascendido a más de 100 millones y en los países en desarrollo 1 de cada 4 personas está en ese período, a diferencia de 1 de cada 7 en los países desarrollados. El embarazo en edades cada vez más tempranas se está convirtiendo en un problema social y de salud pública de alcance mundial. Afecta a todos los estratos sociales, pero predomina en la clase de bajo nivel socioeconómico, en parte debido a la falta de educación sexual y al desconocimiento de los métodos de control de la natalidad. Cuando hablamos de embarazo en la adolescencia nos referimos a la totalidad de embarazos ocurridos en muchachas a una edad cuando aún no son adultas y cuando desde el punto de vista biopsicosocial todavía no están aptas para la maternidad. (Hun Sante , 2000), (Kvause, 1998)

Desde el punto de vista biológico se conoce que hay en la actualidad un desarrollo puberal más temprano en este grupo. Los aspectos psicosociales -de gran importancia- son: inicio precoz de las relaciones sexuales, motivados fundamentalmente por la curiosidad, el deseo sexual y el interés por adquirir experiencias, las que se practican en cualquier lugar donde les sea posible, lo que estimula una conducta sexual irresponsable, que los expone, además, a adquirir infecciones de transmisión sexual. Las adolescentes que se embarazan, además de ver frustrados sus estudios, son solteras en su mayoría, o de lo contrario, inciden posteriormente las separaciones. .Por otra parte, la no utilización de métodos anticonceptivos en la mayoría de las y los adolescentes y la comprensión de todos los riesgos que conlleva el embarazo en estas edades, no siempre es percibido por algunos miembros de la familia con la cual conviven o se relacionan, lo que influye, desfavorablemente, en la prevención del embarazo. (Lugones Botell, 2014)

En ese mismo sentido las adolescentes tienen el doble de probabilidad de morir en relación con el parto que las mujeres mayores de 20 años; en aquéllas por debajo de 15 años de edad aumenta en 5 veces el riesgo. También tienen mayor riesgo de presentar partos distócicos, prolongados, y otras complicaciones, entre las que sobresale la preeclampsia y eclampsia, entre otras. (Lugones Botell, 2014)

El embarazo influye en la vida de la adolescente cuando todavía no alcanza la madurez física y mental, a veces en circunstancias adversas, como son las carencias nutricionales u otras enfermedades. Se ha 
demostrado por numerosos investigadores que a las gestantes que no han completado su crecimiento, se les hace imprescindible una ganancia de peso superior al de la embarazada adulta para lograr un neonato con peso adecuado, tiene una gran repercusión biopsicosocial sobre el organismo de la madre y de su futuro hijo y a medida que ocurre en edades más tempranas esta es más evidente. La mayor cifra de las gestantes adolescentes tiene entre 16 y 17 años. Diversos autores refieren que es frecuente que las adolescentes ignoran los riesgos y daños que pueden significar las relaciones sexuales desordenadas, entre ellos el embarazo. (López Rodríguez, 2012)

Algunos autores denominan el embarazo en la adolescencia como el síndrome del fracaso porque realmente se han frustrado la conducción de la familia, de la escuela y de la sociedad.

El embarazo en la adolescencia es una preocupación de estos tiempos. Estudios realizados por demógrafos cubanos han demostrado un rejuvenecimiento de la fecundidad, con gran incidencia en el grupo de edad comprendido entre 10 y 19 años, fundamentalmente a partir de los 15 años, a ello se asocia el incremento de las tasas de aborto en menores de 20 años, quienes han estado influenciadas por diversas causas que se asocian a una deficiente educación sexual, tanto en el hogar como en las instituciones educacionales de salud. Sarmiento encontró una alta incidencia de embarazos, la mayoría no deseados, en su estudio con adolescentes.

La fecundidad total y la del grupo de 15 a 19 años han disminuido en la región de las Américas. Sin embargo, estos descensos no han sido de igual magnitud en todos los grupos etarios, ni en todos los países. La tasa de fecundidad específica en el grupo de 15 a 19 años en América Latina fluctúa entre 60 y 130 nacimientos por 1.000, tendiendo, en general, las tasas de fecundidad en adolescentes a ser más altas en los países de fecundidad alta. (López Rodríguez, 2012)

Las investigaciones sobre la salud sexual y reproductiva en la adolescencia, han estado dirigidas tradicionalmente a problemas relacionados con la reproducción, específicamente el embarazo y la maternidad. Menos atención se ha brindado a los problemas relacionados con la sexualidad y por consiguiente existe una ausencia de información desagregada por sexo sobre estos aspectos y su impacto en la salud y las vidas de los adolescentes. La salud de los adolescentes (10-19 años), es un 
elemento clave para el progreso social, económico y político de todos los países y territorios, sin embargo, con demasiada frecuencia, las necesidades y los derechos de los adolescentes no figuran en las políticas públicas, ni en la agenda del sector salud excepto cuando su conducta es inadecuada. (López Rodríguez, 2012)

Hoy día es muy importante abordar la temática de la adolescencia y la juventud, debido a la trascendencia de este grupo etáreo para el desarrollo y a su impacto en la población mundial, particularmente en América Latina y el Caribe, donde la población comprendida entre 10 y 24 años de edad representa un $30 \%$ y los adolescentes entre 11 y 19 años, un $20 \%$, con una distribución equitativa entre ambos sexos.

\section{Desarrollo}

La Organización Mundial de la Salud considera que el embarazo durante la adolescencia es un embarazo de riesgo, debido a las repercusiones que tiene sobre la salud de la madre y el feto, además de las secuelas psicosociales, particularmente sobre el proyecto de vida de los adolescentes.

Tener un hijo en la adolescencia dependerán del contexto particular en que se desarrolle esta situación y por ello, más que un problema en sí mismo, el embarazo en la adolescencia debe entenderse en función de los múltiples procesos a los que está asociado y a las significaciones que éste adquiere en cada cultura y grupo social. (Vélez Arango, 2012)

El proyecto de vida y el embarazo en el adolescente se identifican de manera general dos patrones que muestran realidades diferenciadas con relación al embarazo en la adolescencia. Por un lado importantes sectores que viven una realidad sociocultural y económica en la que el embarazo adolescente no necesariamente es conceptualizado como una problemática por los individuos o sus comunidades y, más bien forma parte de las prácticas culturales, o es una forma para las mujeres de constituir su propio proyecto de vida en contexto socioeconómicos caracterizados por la falta de oportunidades. Por otro lado, está un patrón que se registra en contextos urbanos con una importante proporción de embarazos adolescentes no planeados/ deseados 
y en situación premarital, en las que el embarazo no responde necesariamente a un proyecto de vida de los individuos, sino que más bien lo interrumpe. (Vélez Arango, 2012)

\section{La salud sexual y reproductiva en los adolescentes.}

La sexualidad y la reproducción están íntimamente ligadas a la calidad de vida, tanto en el ámbito de lo individual como de lo social. La SSR se refiere a un estado general de bienestar físico, mental y social, y no a la mera ausencia de enfermedades o dolencias en todos los aspectos relacionados con la sexualidad y la reproducción, y entraña la posibilidad de ejercer los derechos sexuales y reproductivos.

La sexualidad hace parte normal del desarrollo del adolescente. Ella es fuente de comunicación y se expresa principalmente en tres áreas: la genital o biológica que se expresa en los aspectos fisiológicos, el área erótica -relacionada con la búsqueda de placer, y la moral expresada en la estructura social fijados en los valores aceptados.

La esfera de la sexualidad introduce a los adolescentes en un conjunto de escenarios de riesgo, frente a los cuales toman decisiones de acuerdo con determinadas concepciones sobre la salud y la enfermedad, sobre el cuerpo y sobre los valores que le inculcó la sociedad. En la experiencia diaria puede observarse que hay adolescentes que pueden ser perfectamente conscientes de los riesgos y conocen las consecuencias de ser activos sexualmente, así como otros que desconocen por completo. Es así como este grupo necesita servicios de consejería en SSR para que puedan aclarar las preguntas sobre las formas de evitar embarazo no deseado, y de cómo disminuir los riesgos de adquirir una ITS, incluida el VIH/SIDA.

La salud sexual se evidencia en las expresiones libres y responsables de capacidades sexuales que conducen a la armonía personal y al bienestar social, enriqueciendo la vida individual y social

El desarrollo completo de la sexualidad es esencial para el bienestar individual, interpersonal y social, e incluye temas como sexo, género, identidad sexual y de género, orientación sexual, erotismo, apego emocional, amor y reproducción. 
Se experimenta o se expresa en pensamientos, fantasías, deseos, creencias, actitudes, valores, actividades, prácticas, funciones y relaciones.

La salud reproductiva se refiere al bienestar físico, mental y social de mujeres y hombres en asuntos relacionados con el sistema reproductivo y sus funciones. (Rodríguez Gázquez, 2008)

(Mendoza Tascón, Claros Benítez, \& Peñaranda Ospina, 2016) En su artículo hace referencia a algunos determinantes de riesgo para el inicio de la actividad sexual temprana en la adolescencia, exponemos a continuación:

Determinantes individuales.

- Sexo masculino

- Actitud permisiva hacia la AS en la adolescencia

- Consumo de licor

- Consumo de tabaco

- Consumo de droga

Determinantes familiares

- Falta de educación sexual por parte de la madre o la poca comunicación en los temas referidos a la sexualidad

- Conductas disciplinarias extremistas

- Relación nulo o pobre entre los padres y los adolescentes

- Mayor escolaridad de los padres.

Determinantes socio-culturales y políticos

- Pertenecer a estratos socioeconómicos bajos

- Influencia del ambiente y contexto social

- Pobre o nula planificación familiar en colegio o servicio de salud

- Falta de políticas publicas 
- Erotización de los medios de comunicación. (Mendoza Tascón, Claros Benítez, \& Peñaranda Ospina, 2016)

Resumiendo, la aprobación de las relaciones sexuales por parte de adolescentes se ve influenciada por su ambiente y contexto social, comportamiento par o amigo y percepción que tienen los adolescentes de la presión existente por parte de sus pares y de sus amigos para tener o no relaciones sexuales a su edad. No obstante, el apoyo mutuo para no tener sexo, es un factor protector para el inicio temprano de la AS. Brendgen y cols, hallaron que el rechazo de los pares se asocia indirectamente con un riesgo más elevado de AS temprana por su vinculación con la baja autoestima, pero sólo para las niñas. Mientras tanto, el abuso verbal por los profesores durante la infancia se asoció directamente con un mayor riesgo de tener AS temprana. (Brendgen, Wanner, \& Vitaro, 2007)

Diversos determinantes han sido implicados con el embarazo en adolescentes, y si bien muchos adolescentes tal vez deseen quedar embarazadas, muchos se producen en el contexto de violaciones de derechos humanos como el matrimonio infantil, relaciones sexuales forzadas o el abuso sexual. Este fenómeno se ha vinculado a múltiples determinantes individuales, familiares, socioculturales y políticos. Hasta un $49 \%$ de adolescentes en embarazo se encuentran por fuera de escuelas y colegios, otras abandonan los estudios secundarios y 7,5\% lo hacen por estar en embarazo y $5 \%$ por que se casan, entre otras razones. Las mujeres sin escolaridad tienen la edad de la primera unión marital a los 17,7 años. Un $81 \%$ de adolescentes ha usado un método anticonceptivo alguna vez en su vida, pero sólo $10 \%$ los usa en su primera relación sexual, siendo este uno de los determinantes más importantes para embarazos precoces. (Chandra-Mouli \& Michaud, 2013) (Sánchez, Grisales, Ceballos, Bustamante, Muriel, \& al., 2013)

Es esencial agregar que las mujeres adolescentes embarazadas están más predispuestas a complicaciones, como la hipertensión inducida por el embarazo, la preclamsia, la eclampsia, la poca ganancia de peso, la anemia, el parto pre término, las hemorragias, el trabajo de parto prolongado, las lesiones durante el parto y la desproporción céfalo pélvica, así mismo, la gestante adolescente tiene mayor riesgo de sufrir violencia y abuso sexual, recurrir a métodos peligrosos para abortar, retraso en 
la atención de complicaciones derivadas del aborto por miedo a la censura aumentando el riesgo de enfermedad y muerte, y mayor número de hijos al final de su vida reproductiva . (Bello-Carrasco , Domínguez-Olmedo, \& Mera-Espinoza, 2016), (Manrique , 2008)

\section{Embarazo en la adolescencia. Ecuador.}

Ecuador es el tercer país con la tasa más alta de embarazos adolescentes de la región. En los últimos 10 años, el incremento de partos en adolescentes de entre 10 y 14 años fue del $78 \%$. Guayas, Pichincha, Manabí, Los Ríos y Esmeraldas son las provincias que concentran el mayor número de embarazos adolescentes en el país. Entre el 2014 y 2016 se produjeron cinco embarazos diarios de menores de 14 años en este país, existiendo algunos casos de violencia sexual y en otros casos de embarazos tempranos que son consensuados con la pareja.

El Plan Nacional de Salud Sexual y Reproductiva para el período 2017- 2021 y la implementación de Estrategia Nacional Intersectorial de Planificación Familiar y Prevención del Embarazado Adolescente (Enipla) tiene como objetivo aplicar la política del control del embarazo adolescente. Esto indica los esfuerzos que hacen las diferentes organizaciones internacionales y el país para prevenir el embarazado en las adolescentes y la necesidad de una adecuada preparación de estos para evitar riesgo en la salud sexual y personal; teniendo objetivo priorizado: “. . la atención a la sexualidad desde las primeras edades, combatir el sexismo y la discriminación de género en la sociedad ecuatoriana y promover, a través del sistema educativo, la equidad entre mujeres y hombres. (Ministerio de Educación en Ecuador , 2013), (Demera Muentes \& Lescay Blanco, 2017), (Embarazo adolescente, un problema social en Ecuador. , 2018.)

A nivel nacional, la reducción de la mortalidad materna - perinatal es una prioridad y se refleja en las metas 3 y 4 del objetivo 3 del Plan Nacional del Buen Vivir que es el documento que guía el hacer de las políticas públicas del Ecuador

El cantón Manta de la provincia de Manabí, se encuentra constituido por: barrios, ciudadelas, parroquias y comunidades. Entre las características más notables que conforman estas áreas geográficas se destacan: 
- Continúa migración de pobladores de la zona rural de toda la provincia.

- Bajos recursos económicos.

- Niveles bajos de escolaridad,

- La población manabita presenta como tradición una conducta machista entre los hombres y la cultura de la sociedad de la creencia de tal forma de conducta social.

Estos discernimientos, entre otros, son causales que promueven la falta de educación preventiva, por parte de la familia, hacia la(o) s adolescentes; que trae como consecuencia, las conductas poco responsables de los sujetos ante las necesidades y relaciones sexuales, caracterizada por la falta de protección, los embarazos no deseados, enfermedades de transmisión sexual, abortos provocados, partos prematuros, acompañado de la deserción escolar. (Prevención del embarazo precoz en adolescentes desde la perspectiva de la enfermería en la ciudadela Eloy Alfaro de Manta del 2014 al 2016. )

En el Ecuador muchas han sido las investigaciones sobre el embarazo precoz en la adolescencia y su incidencia en la salud, han analizado el embarazo precoz en las adolescentes mujeres que se encuentran cursando en colegio y su incidencia en el aprendizaje, también han abordado la temática desde la prevalencia y factores de riesgo que están asociados a embarazos en adolescentes que acuden a consulta externa de los hospitales.

En la Universidad Laica Eloy Alfaro de Manabí se realizó un proyecto Prevención del embarazo precoz en adolescentes desde la perspectiva de la enfermería en la Parroquia Eloy Alfaro de la ciudad de Manta del 2014 al 2016. El resultado del proyecto fue: Débil nivel de educación sexual y educativa en adolescente, trayecto como consecuencias:

- Grupo de riesgo no identificado

- Población con problemas sociales culturales y económicos

- Comunidad con cierto grado de insatisfacción con los programas del Ministerio de Salud Pública. (García , Santos , \& Riofrio, 2016.) 
Se realizó un estudio por parte de (Demera Muentes \& Lescay Blanco, 2017), donde se aplicó una entrevista para los estudiantes de primer año de bachillerato general unificado en la Unidad Educativa Fiscal "Olmedo" de la ciudad de Portoviejo, con el objetivo de constatar su conocimiento acerca del embarazo precoz y la sexualidad, donde se comprobaron los siguientes resultados .

1. Desconocimiento por parte del adolescente sobre el embarazo

2. Insuficiente papel de la familia y la escuela en correspondencia con la orientación en la sexualidad 3. Las adolescentes consideran necesario una educación sexual que las oriente y le fortalezca los modos de comportamiento sexuales y sociales.

4. Existencia de tabúes en la familia con respecto a los temas de la sexualidad

5. Necesidad de que la institución educativa promocione acciones preventivas dirigidas al embarazo precoz en adolescentes.

De manera semejante Ubillus Saltos S P, Zambrano Santos R O, Sánchez Rodríguez J M, Sánchez Rodríguez L. (2016) realizaron una investigación acerca de la prevención educativa del embarazo precoz del estudiante del Nivel Básico Superior de la Unidad Educativa de Portoviejo. Manabí, evidenciando la insuficiente educación de la sexualidad en estudiantes de este nivel y justifica la necesidad de la construcción de un modelo educativo y preventivo del embarazo precoz, desde la acción tutorial, en calidad de contribución a la teoría pedagógica. (Ubillus Saltos, Zambrano Santos, Sánchez Rodriguez, \& Sánchez Rodríguez, 2016)

(Bello-Carrasco , Domínguez-Olmedo, \& Mera-Espinoza, 2016) Arrojaron en su investigación que el embarazo no deseado en los estudiantes del cantón de Manta afecto su nivel emocional.

En otro escenario de la comunidad universitaria se realizó una investigación con los estudiantes de segundo semestre de la Escuela Superior Politécnica de Chimborazo (ESPOCH) teniendo como resultado vulnerabilidad en la salud sexual y reproductiva de los jóvenes universitarios. (Saeteros Hernández, Pérez Piñero, \& Sanabria Ramos, 2015) 
Entre los expertos de la temática existe consenso que una adecuada educación sexual contribuye positivamente a la disminución en la frecuencia de actividad sexual precoz.; lo que reafirma la necesidad imperiosa de desarrollar programas destinados a fortalecer las habilidades y capacidades de los adolescentes para de esta forma evitar embarazos en esta etapa temprana de la vida que conlleve en la mayoría de los casos a la discapacidad infantil.

Es sustancial encomendar que se identifiquen los riesgos potenciales de embarazo precoz e infecciones de transmisión sexual, a través de la interacción escuela-familia-consultorio y establecer vínculos que permitan planificar, organizar y ejecutar programas de educación sexual en las escuelas y la comunidad donde la adolescente se apropie de conocimientos esenciales para prevenir el embarazo, así como la presencia de complicaciones potencialmente discapacitantes.

\section{Conclusión}

Desde la educación de la sexualidad la prevención del embarazo precoz en los adolescentes constituye una necesidad para su cuidado de la salud de manera que puedan atenuar los factores de riesgo asociados al embarazo. Resultando acogimiento de conductas responsables ante la salud como condición para lograr el empoderamiento en promoción de salud.

\section{Referencias Bibliográficas}

Embarazo adolescente, un problema social en Ecuador. . ( 2018.). Obtenido de https://www.publicafm.ec/noticias/ecuador/1/embarazo-adolescente-ecuador

American Social Health Association. (Asociación Estadounidense de Salud Social). . (2009). Obtenido de http://www.iwannaknow.org/

Bello-Carrasco , L. M., Domínguez-Olmedo, E., \& Mera-Espinoza, G. (2016). El embarazo en las adolescentes estudiantes del cantón Manta. Revista científica . Dom. Cien 2 (4) pag 40-50.

Brendgen, M., Wanner, B., \& Vitaro, F. (2007). Peer and teacher effects on the early onset of sexual intercourse. Am J Public Health 2007;97:2070-5. 
Centre for Disease Control and Prevention (CDC). (s.f.). Sexually Transmitted Disease Surveillance 2005 Supplement, Syphilis Surveillance Report. Diciembre de 2006. Obtenido de http://www.cdc.gov/std/syphilis2005/syphsurvsupp2005short.pdf.

Chandra-Mouli , V., \& Michaud, P. A. (2013). WHO guidelines on preventing early pregnancy and poor reproductive outcomes among adolescents in developing countries. J Adolesc Health ;52

Demera Muentes, F. M., \& Lescay Blanco, D. M. (2017). La prevención del embarazo en los adolecentes de la educación general básica en el Ecuador . .

García , E., Santos , M., \& Riofrio, M. I. (2016.). PROYECTO Prevención del embarazo precoz en adolescentes desde la perspectiva de la enfermería en la Parroquia Eloy Alfaro de la ciudad de Manta del 2014 al 2016.

Hun Sante , S. M. (2000). El embarazo en la adolescencia precoz. Instantáneas. Rev Panam Salud Pública ; 4(2):262-3.

Kvause, M. (1998). Algunos temas fundamentales de educación sexual. Embarazo en la adolescencia. La Habana: Editorial Científico-Técnica.

López Rodríguez, Y. (2012). Embarazo en la adolescencia y su repercusion biopsicosocial sobre el organismo de la madre y de su futuro hijo .Revista Cubana de Enfermeria ,28(1),23-36. Obtenido de http://scielo.sld.cu/scielo.php?script=sci_arttext\&pid=S086403192012000100004\&lng=es\&tlng=es.

Lugones Botell, M. (2014). Embarazo en la adolescencia, importancia de su prevención en la Atención Primaria de Salud.Revista Cubana de Medicina General Integral ,30(1),1-2. Obtenido de http://scielo.sld.cu/scielo.php?script=sci_arttext\&pid=S086421252014000100001\&lng=es\&tlng=es

Manrique, R. (2008). Parto pretérmino en adolescentes. Rev de Obstet Ginecol Venez; 68(3): p. 141143.

Mendoza Tascón, L. A., Claros Benítez, D. I., \& Peñaranda Ospina, C. B. (2016). Actividad sexual temprana y embarazo en la adolescencia: estado del arte. Revista chilena de obstetricia y ginecología, 81(3), 243-253. Obtenido de https://dx.doi.org/10.4067/S071775262016000300012 
Ministerio de Educación en Ecuador . (2013). Educación de la sexualidad y afectividad. Guía para formadores. Quito. Ecuador. .

Prevención del embarazo precoz en adolescentes desde la perspectiva de la enfermería en la ciudadela Eloy Alfaro de Manta del 2014 al 2016. . (s.f.). Obtenido de http://departamentos.uleam.edu.ec/vinculacion-colectividad/files/2015/10/Ver-Proyecto2.pdf

Rodríguez Gázquez, M. (2008). Factores de riesgo para embarazo adolescente. Medicina U.P.B., 27 (1), 47-58.

Saeteros Hernández, R. d., Pérez Piñero, J., \& Sanabria Ramos, G. (2015). Conducta de riesgo y problemas sexuales y reproductivos de estudiantes universitarios ecuatorianos. Humanidades Médicas, $\quad$ 15(3), 421-439. Obtenido de http://scielo.sld.cu/scielo.php?script=sci_arttext\&pid=S1727-

$81202015000300003 \& \operatorname{lng}=\mathrm{es} \& \operatorname{tlng}=\mathrm{es}$

Sánchez, Y. A., Grisales, M. B., Ceballos, L. Y., Bustamante, J. C., Muriel, E., \& al., e. (2013). Características pobla-cionales y factores asociados a embarazo en mujeres adolescentes de Tuluá, Colombia. Rev Chil Obstet Ginecol ; 78(4): 269-281.

Sexo y Salud,2005. (s.f.). Sexo y Salud. La historia de la Anticoncepción. (manual en Línea) 2005/agosto/04. Obtenido de :http://www.dnp.gov.co/archivos/documentos/DEPP_Evaluaciones_Focalizadas/Mod_4_ss.p df.

Ubillus Saltos, S. P., Zambrano Santos, R. O., Sánchez Rodriguez, J. M., \& Sánchez Rodríguez, L. (2016). Prevención educativa del embarazo precoz del estudiante del nivel básico superior.

Vaillant Correoso, M., Dandicourt, T. C., \& Mackensie, S. Y. (2012). Prevención del embarazo en adolecente.Rev Cubana Enfermeria, 28(2), pp 125-135 . Obtenido de http://scielo.sld.cu/scielo.php?script=sci_arttext\&pid=S0864-03192012000200008\&lng=es.

Vélez Arango, A. L. (2012). El embarazo en el adolescente: una visión desde la dimensión emocional y la salud pública Revista Cuidarte, 3( 1), ENERO-DICIEMBRE, 2012, PP. 394-403 Universidad de Santander Bucaramanga. Colombia. 